\title{
Analysis of STEM Majors' Calculus Knowledge by Using APOS Theory on a Quotient Function Graphing Problem
}

\section{Dr. Emre Tokgoz, Quinnipiac University}

Emre Tokgoz is currently an Assistant Professor of Industrial Engineering at Quinnipiac University. He completed a Ph.D. in Mathematics and a Ph.D. in Industrial and Systems Engineering at the University of Oklahoma. His pedagogical research interest includes technology and calculus education of STEM majors. He worked on an IRB approved pedagogical study to observe undergraduate and graduate mathematics and engineering students' calculus and technology knowledge in 2011. His other research interests include nonlinear optimization, financial engineering, facility allocation problem, vehicle routing problem, solar energy systems, machine learning, system design, network analysis, inventory systems, and Riemannian geometry. 


\title{
Analysis of STEM Majors' Calculus Knowledge by Using APOS Theory on a Quotient Function Graphing Problem
}

\author{
Emre Tokgöz \\ Emre.Tokgoz@qunnipiac.edu \\ Department of Engineering, School of Business \& Engineering, Quinnipiac University, Hamden, CT, 06518
}

\begin{abstract}
Success in many engineering and mathematics courses is tied to well-developed calculus knowledge. Several important calculus concepts used in STEM courses include limit, first derivative, second derivative, and asymptote. In this article, undergraduate and graduate engineering and mathematics students' ability to transform an algebraic function to its geometric representation is analyzed. Participants were either enrolled or recently (two week period) completed a Numerical Methods/Analysis course during the data collection period. Video recorded and written responses to graphing a quotient function are analyzed by using Action-ProcessObject-Schema (APOS) theory. Participants are asked to sketch the graph of the given quotient function after calculating its limiting values, first derivative, second derivative and asymptotes. Qualitative and quantitative results indicated Mathematics majors' higher success rate among all the participants.
\end{abstract}

Key words: APOS theory, Schema, Triad Classification, Functions, Derivative, Limit, Asymptote, Critical Points.

\section{Introduction}

Engineering majors' calculus knowledge development is one of the main goals of several engineering university curriculums. Function concept is particularly important in engineering education because of its ties to many other calculus topics such as limits, derivatives, and integrals. Therefore, understanding STEM majors' ways of viewing and recalling calculus concepts has been an important task of researchers interested in STEM education. (see for example Baker, Cooley, and Trigueros (2000) and Cooley, Trigueros, and Baker (2007)) This is due to the fact that students need to recall and apply calculus concepts in advanced courses. Numerical Methods/Analysis is an example of such advanced level courses in which calculus concepts are frequently used. Therefore, it is natural to measure senior undergraduate and graduate STEM majors' calculus sub-concept knowledge. Action-Process-Object-Schema (APOS) theory will be used to find a qualitative and quantitative answer to our goal in this work.

Baker, Cooley, and Trigueros (2000) analysis of students' conceptual calculus understanding was based on a calculus graphing problem in which several conditions are required to be satisfied. In 2007, Cooley, Trigueros, and Baker conducted a more detailed study than in their research in 2000 to observe calculus concept knowledge of students who were considered to be successful by their professors in diverse disciplines, and by using the same theoretical framework of their study in 2000.

The motivation behind this study is to analyze conceptual calculus knowledge of the undergraduate and graduate students' who were enrolled in a Numerical Methods or Analysis course by using a theoretical framework similar to that of Baker et al. (2000) and 
Cooley et al. (2007) In this study a quotient function is given in its algebraic form and participants are asked to employ their calculus knowledge to determine the graph of the corresponding function. Written and video recorded oral interview responses of participants are analyzed by using the APOS theory.

\section{APOS Theory and Literature}

The philosophy of mathematics influenced researchers in mathematics and engineering education in the undergraduate curriculum in the 1990s. Piaget's schemes idea in the 1970's, and its development with detailed explanations by Piaget and Garcia in the 1980's, influenced researchers of undergraduate mathematics education curriculum in the 1990's. Students' conceptual view of the function was defined by Breidenbach, Dubinsky, Hawks, and Nichols in 1992, which relied on Piaget's study of functions in 1977 (Piaget, Grize, Szeminska \& Bang, 1977). This formed the action-process-object idea in mathematics education for the undergraduate curriculum. In 1996, Asiala, Brown, DeVries, Dubinsky, Mathews, and Thomas added schema idea to the action-process-object idea and formed action-process- object-schema theory (called APOS theory) to mathematical topics (mostly functions), and they explained this theory as the combined knowledge of a student in a specific subject based on Piaget's philosophy. Dubinsky and McDonald (2001) explain the components of the APOS theory as follows:

Action: A transformation of objects perceived either explicitly or from memory that depends on step-by-step instructions on how to perform the operation...

Process: When an action is repeated and the individual reflects upon it, he or she can make an internal mental construction called a process which the individual can think of as performing the same kind of action, but no longer with the need of external stimuli...

Object: An object is constructed from a process when the individual becomes aware of the process as a totality and realizes that transformations can act on it...

Schema: Individuals collection of actions, processes, objects, and other schemas which are linked by some general principles to form a framework in individual's mind...

In APOS theory, concepts are constructed on different concepts and schemas. For example, if a researcher works on the Taylor series expansion of a function, the researcher can base the schemas on understanding functions, limits, derivatives, infinite, continuity, integral, summation, and number knowledge of students. All schema combinations can form a schema. We can also say that every concept requires concept knowledge and the construction of a specific concept depends on knowledge of the other concepts. APOS theory applicable is not necessarily a good method to analyze data as experienced by Clark, Cordero, Cottrill, Czarnocha, DeVries, St. John, Tolias, and Vidakovic (1997) in their research. Piaget et al. (1983) introduced the triad stages intra, inter, and trans, used by Baker et al. (2000), to introduce the property and interval schemas to analyze undergraduate students conceptual function knowledge on a calculus graphing problem. In 2007, Cooley, Trigueros, and Baker built on their work from 2000 (Baker et al. (2000)) by focusing on the thematization of the schema with the intent to expose those possible structures acquired at the most sophisticated stages of schema development. In their study, the problems were structured in a way that participants were required to respond to the first eight questions and 
continue with the ninth question only if they succeeded in answering the first eight questions (please see Cooley et al. (2007) and the appendix, pg. 391 for further details). The detailed analysis of the collected data indicated participants' success in answering a complex graphing problem, thus schema thematization was possible in their study. Cortés (2004) also observed student difficulties in understanding the function concept by using a questionnaire similar to that of Cooley et al. (2007), with similar results. The questionnaire developed in this study contains different questions than that of Cooley et al. (2007) including analytical calculus concept calculations for a quotient function, answering fill-inthe-blank calculus concept questions, and sketching the graph of a function after calculating calculus concept questions.

In the last decade, APOS theory is widely used in several educational research areas. It is used by Parraguez and Oktac (2010) to lead the students towards constructing the vector space concept, by Mathews and Clark (2007) to observe mean, standard deviation, and the central limit theorem knowledge of successful students who completed an elementary statistics course with a grade of "A", by Kashefi, Ismail, and Yusof (2010) to observe students' obstacles in the learning of two variable functions in calculus, and by Tziritas (2011) to observe students' success in solving related rate problems.

\section{Functions \& Calculus in Research on Undergraduate Mathematics Education (RUME)}

The conceptual knowledge of a student is scaled by the researchers based on the student's ability to construct concept-related graphs (conceptual image) and to answer the corresponding algebraic questions. Students' difficulties with the conceptual image are observed by several researchers (Orton, 1983; Selden, Selden, \& Mason, 1994). The derivative and its corresponding graph can have an important role in understanding the graph of the corresponding function. Aspinwall, Shaw, and Presmeg (1997) collected data by observing a student and concluded that incorrectly created derivative images can result in mistakes of analytical reasoning of the student. Given the graph of a function, FerriniMundy and Graham (1994) observed participating students' difficulty in sketching the derivative graph of the given function where many students first tried to find an algebraic representation of the given function. Thompson (1994) observed that senior mathematics undergraduate and graduate students' weak rate of change concept knowledge resulted in weak understanding of the integration concept. Trigueros and Martinez-Planell (2009) and Kashefi et al. (2010) observed students' ability to construct and develop two variable functions by using APOS theory. Kashefi et al. (2010) concluded that in two variable calculus settings students had difficulty in domain, range, and the graphs of two variable functions. 


\section{Methodology}

\section{Participants and the General Procedure}

Seventeen senior undergraduate and graduate students from Engineering and Mathematics disciplines who were enrolled to one of these two courses at a large Midwestern university participated in this study. All had completed multi-variable calculus courses that cover the content of the given questionnaire. The data was collected during a semester that the author of this article taught a senior level undergraduate Computer Science Numerical Methods course. Computer Science undergraduate majors were required to complete this course as a requirement of the Computer Science Bachelor of Science degree. During the same semester, the researcher also graded a senior undergraduate/graduate level Numerical Analysis course offered by the Mathematics Department with students enrolled from various science and engineering disciplines. Each participant was required to complete the same questionnaire that consisted of 15 questions, and interviewed for approximately 40 minutes based on his/her responses to the questionnaire questions. The author video recorded all the interviews and designed the interview questions based on the written responses to the questionnaire questions. Interview data collection is standardized across the participants based on their responses. The detailed data collection procedure with the corresponding interview questions for each question will be explained in the corresponding section. The goal of the questionnaire and the interview questions is to analyze participants' ability to respond to algebraic, analytic, and geometric function-related calculus concept questions. Only 4 out of 15 questions were directly related to conceptual function knowledge, with the rest of the questions related to concepts such as derivatives, integrals, power series, and programming preferences. Results regarding the questions that are not covered in this paper are planned to be published elsewhere.

\section{Research Problem}

The question evaluated in this study is designed to observe participants' ability to determine the intervals of increase-decrease, convexity, critical points, horizontal asymptotes and vertical asymptotes of a quotient function to be able to graph it by using all these properties. This problem aims to observe participants' ability to calculate analytical calculus problems and their ability to reflect the obtained information on a graph.

\section{Schema Classification}

A Scheme is an action which is repeated and can be generalized where the actions are derived from sensory-motor intelligence (Piaget, 1971). The coordination of schemes forms 
actions which are logical structures. Combination of systems and schemes can form the scheme (Piaget, 1971). The concept knowledge can be formed in a larger combination of schemes.

The schema classification of Baker et al. (2000) is based on the following triad classification:

- Intra-Interval: Ability to answer questions regarding the independent intervals where the participant can be confused by the union or intersection of other intervals

- Inter-Interval: Ability to answer questions regarding only sub-domains which consists of two or more intervals but not the entire domain.

- Trans-Interval: Ability to answer questions regarding the entire domain.

- Intra-Property: Ability to interpret every analytical property independently one at a time.

- Inter-Property: Ability to interpret two or more analytical properties simultaneously but not all of them together.

- Trans-Property: Ability to interpret all the analytical properties simultaneously.

The schema classification in this work is structured by observing post interview student responses. The data collected in this study suggested following a similar theoretical triad classification to that of Baker et al. (2000). The design of the question and detailed analysis of the post interview student responses suggested a three-level triad classification of the participants for the question considered in this work:

Intra-level: Responses reflected only one analytical property on the right interval on independent intervals. The responses in this category indicate mistakes in application of two or more analytical properties in two or more intervals.

Inter-level: Participants were able to apply one or more analytical properties on the right interval, which may consist of the combination of independent intervals; however, the combination of these intervals does not form the entire domain. The responses in this category indicate application mistakes in only one analytical property on a certain interval.

Trans-level: The participants in this category made no mistake in the application of the analytical properties throughout the entire domain.

For example, a participant is considered to be in the intra-level if the second derivative and the asymptote information are not applied correctly on two or more intervals. This is a result of participant confusion by the union or intersection of other intervals and the failure to interpret every analytical property independently one at a time. If there is only one analytical property application mistake, such as the first derivative information on a certain interval which cannot consist of the union of independent intervals, then the response is categorized as inter-level. Students' trans-level triad classification is based on their ability to answer the question correctly in the entire domain. 


\section{The Question}

The following question is designed to observe participants' ability to transform an algebraic function from to its geometric/graphical representation by calculating the related limit and derivative questions.

A2G Problem: Please draw the graph of $f(x)=\frac{x}{x+1}$ at (e) below by finding and applying each of the following information if they are applicable.

a) Vertical and horizontal asymptotes of $f(x)$ and limiting values of $f(x)$ at the vertical asymptotes if there exists any vertical asymptote.

b) Local maximum, local minimum and inflection points of $\mathrm{f}(\mathrm{x})$.

c) Intervals where $\mathrm{f}(\mathrm{x})$ is increasing and decreasing.

d) Intervals where $\mathrm{f}(\mathrm{x})$ is convex and concave.

e) Please draw the graph of $f(x)=\frac{x}{x+1}$ by using the information you have in parts (a), (b), (c), and (d) if they are applicable.

During the interviews, participants were initially asked to explain their answers briefly to all the parts (a)-(e) of the question and change the written information if it appears to be incorrect. If they made a mistake in one of the parts (a)-(d), participants were asked to answer particular conceptual questions. If the graph was sketched in part (e) with no or partial responses to the parts (a)-(d), these participants were asked to solve parts (a)-(d) during the interview. If there was no correct response to a particular question during the interview, the participant was assumed to lack knowledge of that concept. The following results are obtained from the written questionnaire answers and interviews.

\section{Results}

The collected data is categorized into four: Asymptote, limit, first derivative, and second derivative knowledge. The following table illustrates the post interview triad classification of the participants.

\begin{tabular}{|c|c|c|c|}
\hline A2G Problem & Intra-Level & Inter-Level & Trans-Level \\
\hline Number of Students & 2 & 5 & 10 \\
\hline
\end{tabular}

The details of the triad classification with particular examples of participant responses and the percentage of student success will be covered in the corresponding calculus subconcept section.

\section{Asymptote Knowledge}

As a result of part (a), 35\% (6/17) of the participants could not find the horizontal asymptote. Only one participant, RP 10, could not find the vertical asymptote classified in the inter-level: 
RP 10: Yeah, the first derivative is to show whether the function is increasing or decreasing or have a changing point. I got that asymptote from when the derivative is equal to zero, I mean when it doesn't exist. Then I got these [pointing

$$
\begin{aligned}
& \lim _{x \rightarrow-1^{-}} f(x)=\frac{-1}{0^{-}}=+\infty, \\
& \left.\lim _{x \rightarrow-1^{+}} f(x)=\frac{-1}{0^{+}}=-\infty .\right]
\end{aligned}
$$

Only one student could neither find the vertical nor the horizontal asymptote. 53\% (9/17) of the participants correctly found both vertical and horizontal asymptotes.

\section{Limit Knowledge}

It is well known that the asymptote knowledge and limiting values are directly related to each other. Therefore the limit information

$$
\lim _{x \rightarrow-\infty} f(x), \lim _{x \rightarrow \infty} f(x), \lim _{x \rightarrow-1^{-}} f(x) \text { and } \lim _{x \rightarrow-1^{+}} f(x)
$$

are expected to be used by the participants to sketch a graph. In this question 72\% (14/17) of the participants were able calculate the limiting values $\lim _{x \rightarrow-\infty} f(x)$ and $\lim _{x \rightarrow \infty} f(x)$ correctly. Only RP 9 could not calculate the limiting values $\lim _{x \rightarrow-1^{-}} f(x)$ and $\lim _{x \rightarrow-1^{+}} f(x)$.

Interviewer: And vertical asymptote, horizontal asymptote here for $\frac{x}{x+1}$. Do you remember the definition of vertical asymptote?

RP 9: I would if you reminded me...

Interviewer: And so $\mathrm{x}=1$ is the vertical asymptote... how can we calculate the horizontal asymptote?

RP 9: No I don't.

One of the participants, RP 16, a mathematics graduate student, used a student-based method at the trans-level to explain the way of finding the limiting values:

Interviewer: For horizontal ones?

RP 16: ... you use the BOBO BOTEN EATSDC.

Interviewer: What is that? ... Can you write it?

RP 16: Sure, it is kind of what I learned from my students... [Writes]

$$
\text { BOBO }
$$

BOTEN

\section{EATS DC}

RP 16: So when you are talking about the degrees, [pointing BOBO] bigger on bottom, $y=0$, zero horizontal asymptote, [pointing BOTEN] bigger on top, none. [Pointing EATS] Exponents are the, EATS, same [pointing DC], divide the coefficients.

\section{First Derivative Knowledge}

Two of the participants failed to find the correct first derivative of the function before the interview. For example, RP 9 found the first derivative of $\mathrm{f}(\mathrm{x})$ to be $-x+\frac{x}{x+1}$.

To find the intervals of increase and decrease, the participants calculated the first derivative and observed whether it is bigger than zero or not. Before the interview 29\% $(5 / 17)$ of the participants found the first derivative of the function greater than zero for all real numbers $x$ even though the function is not defined at $x=-1$; however, these participants reflected the correct interval of increase/decrease on their graphs. 
Two of the participants who calculated the first derivative right made a mistake while finding the intervals of increase/decrease:

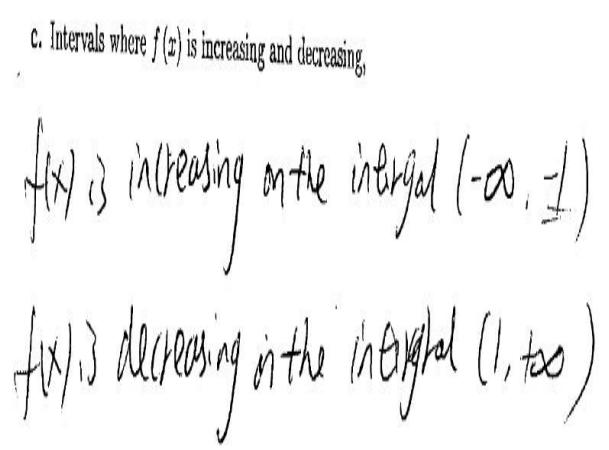

Fig 1. Response of RP 2 to A2G-c.

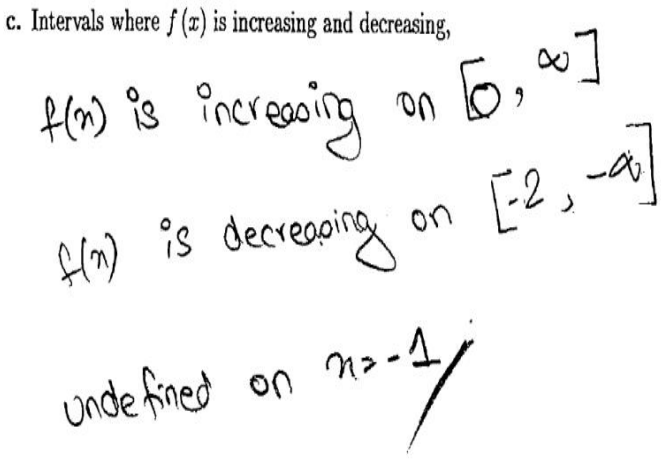

Fig 2. Response of RP 8 to A2G-c.

\section{Second Derivative Knowledge}

$24 \%(4 / 17)$ of the participants miscalculated the second derivative prior to the interview therefore, simple algebraic mistakes played important roles in their resulting graphs:

RP 7: ...I need to take the derivative again. [Starts writing -1] Wait, I don't need to do that. [scratches -1 , starts writing $-(\mathrm{x}+1)^{-2}$.) So [continues writing] taking down a notch. I guess positive three right $\left[\right.$ has $+3(\mathrm{x}+1)^{-}$ ${ }^{3}$ ). I was always bad at these little derivative rules. I always forget them. All the specific derivatives and integrals ...

$88 \%(15 / 17)$ of the participants sketched the convexity information correctly on the graph. Two of these participants sketched the graph of $f(x)=\frac{x}{x+1}$ originating from $\frac{1}{x}$ and two of the participants drew the graph by connecting several dots corresponding to the function on the Cartesian coordinates.

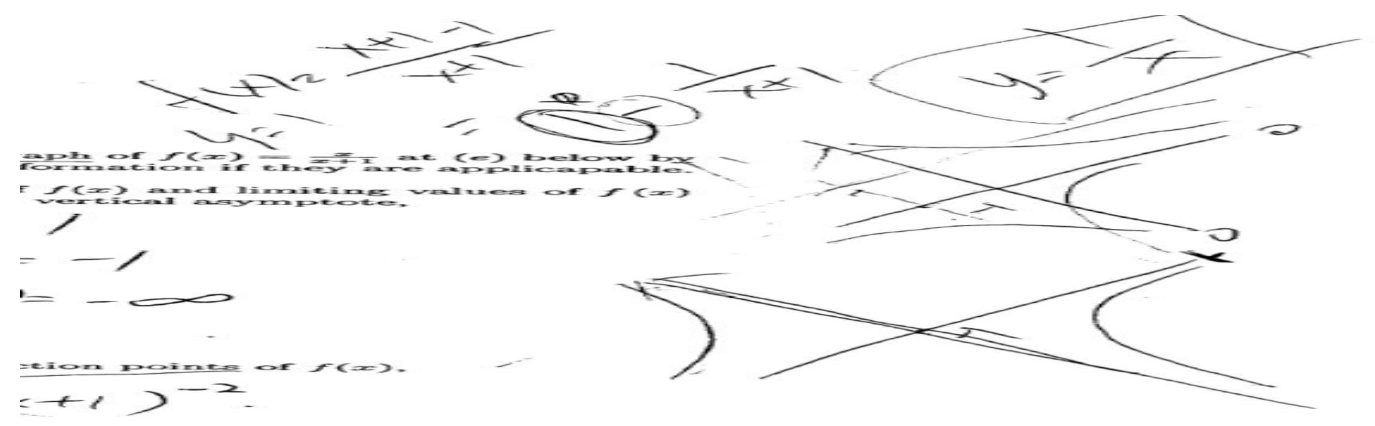

Fig 3. Answer of RP 2 for A2G problem.

RP 14: No, I don't think so. Here are the graphs, and this graph is what I think [pointing $\frac{1}{x}$.] From $\mathrm{y}=\frac{1}{x}$ to $\mathrm{y}=1-\frac{1}{x+1}$ [pointing the graph of $\mathrm{y}=1-\frac{1}{x+1}$ ] 


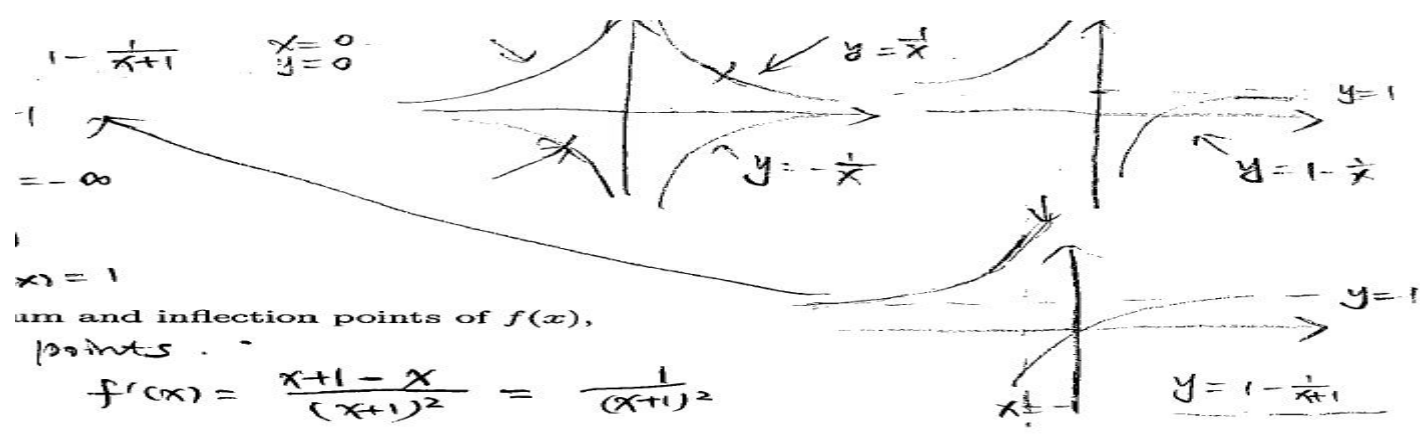

Fig 4. Answer of RP 14 for $A 2 G$ problem.

Interviewer: ... you just... worked out from $\frac{1}{x}$ and you reconstructed the entire graph from there...

These two participants had the correct solutions to the questions (a)-(d) during the interviews.

\section{General Results for A2G Question}

Post-interview data evaluation for the A2G question indicated 59\% (10/17) of the participants could not find the correct interval of increase prior to the interview which indicated the weakness in first derivative knowledge of the participants. Prior to the interview, two of the participants, RP 4 and RP 9, could not calculate the first derivative correctly, and four of the participants could not calculate the second derivative correct, indicating the weakness of participants' algebraic ability to calculate derivatives of a quotient function. Prior to the interview, 6 of the participants showed weakness in horizontal asymptote knowledge and could not reflect it to their graphs. Post-interview results indicated 10 of the participants were successful in sketching the correct graph corresponding to the given function. Baker et al. (2000) reported the lack of conception about the second derivative. The responses to this question also indicate the lack of second derivative knowledge similar to the report of Baker et al. (2000) in addition to the lack of conception about asymptotes. On the contrary to the findings of Slavit (1995), who reported high school honors Algebra II students' difficulty with unfamiliar functions such as functions that are not polynomials, 59\% of the participants succeeded while answering unfamiliar function-related questions.

\section{Results and Discussion}

In this study, we observed the conceptual calculus knowledge of engineering and mathematics undergraduate and graduate students who are either enrolled in or have completed a Numerical Methods or Numerical Analysis course at a large Midwest University. This study is designed to advance the work of Baker et al. (2000) and Cooley et 
al. (2007). Student success while answering calculus concept questions associated with functions is evaluated by using the concept image and concept definition idea of Vinner (1992) and APOS theory with triad classification similar to Baker et al. (2000). The results of this study give insight about Numerical Methods/Analysis students' success in answering several different function-related calculus concept questions. Participants' conceptual calculus concept knowledge is observed by observing the following function question:

A2G Question: Given $f(x)=\frac{x}{x+1}$ students ability to calculate sub-concept information (i.e. limit and derivative information) algebraically and sketch a graph of the function.

Students showed lack of first derivative, second derivative, and limit knowledge success in their responses to the A2G question due to pre-interview responses. Students' encountered difficulty in determining the interval of increase and decrease in this question in addition to determining the horizontal asymptote and reflecting it on the graphs. The first derivative knowledge of the students' appeared to be the major problem in answering this question. Thompson (1994) observed that the rate of change is effective on students' integration. In this study, similar to Thompson's (1994) results, we found the lack of first derivative knowledge affected students function knowledge. Similar to Baker et al. (2000) some of the participants in this study encountered problems with second derivative. Cooley et al. (2007) had a schema thematization in their study; however, because of the complexity of the collected data, a schema thematization is not possible for this study.

Post-interview triad classification for the algebraic expression question indicated translevel classification for most of the participants, and either intra- or inter-levels of classification for most of the other participants. Trans-level categorization for most of the participants is not surprising for engineering and mathematics majors who are expected to have a well-developed background in mathematics.

In relation to the Numerical Methods/Analysis course topics and the expected analytical calculation skills of the students, the algebraic expression question indicated student weaknesses in calculating derivatives. Derivative calculations is an important analytical part of the topics covered in the Numerical Methods/Analysis courses, in particular when the Taylor series expansion of functions are concerned. Therefore, the findings of this study indicate students' weaknesses in analytic derivative calculations, suggesting course instructors should stress derivative concept. Further investigation is necessary to attain a better understanding of participants' conceptual knowledge.

In conclusion, considering the APOS theory-based data classification, post-interview data collection indicated a uniform triad classification of the participants. The responses to the $A 2 \mathrm{G}$ question indicated participant weakness in calculus-concept related analytical calculations of an algebraic function. The main difficulty of the participants appeared to be the first and second derivative calculations. 


\section{References}

1. Asiala, M., Brown, A., DeVries, D. J., Dubinsky, E., Mathews, D., \& Thomas K. (1997). A framework for research and curriculum development in undergraduate mathematics education. In J. Kaput, A. H. Schoenfeld, \& E. Dubinsky (Eds.), Research in collegiate mathematics education II (pg. 1-32). Providence, RI: American Mathematical Society and Washington, DC: MAA.

2. Aspinwall, L., Shaw, K. L., \& Presmeg, N. C. (1997). Uncontrollable men-tal imagery: Graphical connections between a function and its derivative, Educational studies in mathematics, 33, 301-317.

3. Baker, B., Cooley, L., \& Trigueros, M. (2000). A calculus graphing schema, Journal for Research in Mathematics Education, 31(5), 557-578.

4. Breidenbach, D., Dubinsky, E., Hawks, J., \& Nichols, D. (1992). Development of the process of function, Educational Studies in Mathematics, 23(3), 247-285.

5. Clark, J. M., Cordero, F., Cottrill, J., Czarnocha, B., DeVries, D. J., St. John, D., Tolias, G., \& Vidakovic, D. (1997). Constructing a schema: The case of the chain rule?, Journal of Mathematical Behavior, 16, 345-364.

6. Cooley, L., Trigueros M., and Baker B. (2007). Schema thematization: A theoretical framework and an example. Journal for Research in Mathematics Education, 38(4), 370 - 392.

7. Dubinsky, E. \& McDonald, M. A. (2002). APOS: A Constructivist Theory of Learning in Undergraduate Mathematics Education Research, the Teaching and Learning of Mathematics at University Level, 7 (3), 275-282.

8. Ferrini-Mundy, J. \& Graham, K. (1994). Research in calculus learning: Understanding limits, derivatives, and integrals. In E. Dubinsky \& J. Kaput (Eds.), Research issues in undergraduate mathematics learning, 19-26. Washington, DC: Mathematical Association of America.

9. Kashefi H., Ismail Z., \& Yusof, Y. M. (2010). Obstacles in the Learning of Two-variable Functions through Mathematical Thinking Approach, International Conference on Mathematics Education Research, Social and Behavioral Sciences, 8, 173-180.

10. Mathews, D., \& Clark, J. (1997). Successful students' conceptions of mean, standard deviation, \& the Central Limit Theorem. Presentation at the Midwest Conference on Teaching Statistics, WI.

11. Orton, A. (1983). Students' understanding of differentiation, Educational Studies in Mathematics, 14, 235-250.

12. Parraguez, M. \& Ortac, A. (2010). Construction of the vector space concept from the viewpoint of APOS theory, Linear Algebra Appl. 432 (8), 2112-2124.

13. Piaget, J. (1971). Psychology and epistemology (A. Rosin, Trans.). London: Routledge and Kegan Paul. (Original Work Published 1970)

14. Piaget, J., J.-B. Grize, A., Szeminska, and Bang, V. (1977). Epistemology and psychology of functions ( J. Castellano's and V. Anderson:Trans.)

15. Piaget, J., \& Garcia, R. (1989). Psychogenesis and the history of science (H.Feider, Trans.). New York: Columbia University Press. (Original work published in 1983).

16. Selden, J., Selden, A., \& Mason, A. (1994). Even good calculus students can’t solve non-routine problems. In E. Dubinsky \& J. Kaput (Eds.), Research issues in undergraduate mathematics learning (31-45). Washington, DC: Mathematical Association of America.

17. Slavit, D. (1995). A growth-oriented route to reification of function. In D. T. Owens, M. K. Reed, \& G. M. Millsaps (Eds.), Proceedings of the seventeenth annual meeting of the North American Chapter of the International Group for the Psychology of Mathematics Education (Vol. 1, pp. 284-290). Columbus, OH: ERIC Clearinghouse for Science, Mathematics, and Environmental Education.

18. Thompson, P. W. (1994). Students, functions, and the undergraduate curriculum, Conference Board of the Mathematical Sciences Issues in Mathematics Education, 4, 21-44.

19. Trigueros, M., \& Martínez-Planell. R. (2009). Geometrical Representations in the lLearning of Twovariable Functions. Educ Stud Math, Published online, 24 June.

20. Vinner, S. (1992). The function concept as a prototype for problems in mathematical learning. In E. Dubinsky \& G. Harel (Eds.), the concept of function: Aspects of epistemology and pedagogy (195213). Washington, DC: MAA. 